\title{
HYPERONIC CRYSTALLIZATION IN HADRONIC MATTER
}

\author{
M.A. PÉREZ-GARCÍA ${ }^{1}$ \\ E-mail: aperez@pinon.ccu.uniovi.es \\ J. DÍAZ-ALONSO ${ }^{1,2}$, L. MORNAS ${ }^{1}$, J.P. SUÁREZ ${ }^{1}$ \\ $\left(^{1}\right)$ Dpto. de Fisica, Universidad de Oviedo. Avda. Calvo Sotelo 18, E-33007 \\ Oviedo, Asturias, Spain \\ $\left(^{2}\right)$ DARC, Observatoire de Paris - Meudon, F-92195 Meudon, France
}

\begin{abstract}
The possible formation of a spatially ordered phase in neutron star matter is investigated in a model where hyperonic impurities are localized on the nodes of a cubic lattice.
\end{abstract}

We propose a method for analyzing the formation of spatially ordered configurations in hadronic matter, related to the crystallization of the hyperonic sector in a lattice. The method allows the determination of the density ranges where such ordered configurations are energetically favoured with respect to the usual gaseous configurations. This leads to the determination of the parameters of a first order phase transition from the fluid to the crystallized state as well as the equation of state of the plasma in the ordered phase. We show some preliminary results obtained by the application of the method to a very simplified model which shows the efficiency of the mechanism of confinement of the hyperons in an ordered phase proposed here. The eventual existence of such a new phase should induce changes in the structure and cooling of dense stars.

At densities about 1.5 times nuclear saturation and beyond, hyperons are present in hadronic matter. The equation of state taking into account the presence of nucleons and hyperons which interact through the exchange of several mespns has been extensively studied using phenomenological lagrangian models 1 . In the mean field approximation, the hyperonic component (as the other hadronic components in the ground state) is supposed to form a spatially uniformly distributed Fermi fluid undergoing the action of the mesonic mean field created by the whole baryonic distribution in the plasma.

The hadronic interaction in our model is reduced to the exchange of scalar and vector meson fields. Such a description is the simplest one allowing for an acceptable fit of nuclear saturation 2 . We consider a unique species of neutral hyperons. The interaction Lagrangian for every baryon species $B$ reads

$$
L_{I}=\sum_{B=p, n, h} g_{\sigma B} \bar{\Psi} \sigma \Psi+g_{\omega B} \bar{\Psi} \gamma^{\mu} \omega_{\mu} \Psi
$$


where the coupling constants to the meson fields are weaker for hyperons than for nucleons. The leptonic sector is reduced for simplicity to the electron.

Using relativistic condensed matter techniques, we investigate the possibility of the existence of energetically favourable solid configurations, where pairs of antiparallel spin hyperons are confined on the nodes of a regular lattice in the ground state of the hadronic plasma. In this situation the hyperons should behave as impurities which induce a redistribution of the surrounding nucleons. Nevertheless, as a first approximation to the solution for the dynamics of the nucleon in the medium, we consider the lattice of neutral hyperons as a uniform background, in an analogous way to the "free electron model" in a Coulomb lattice 6 .

In evaluating the energetic contribution of a crystal array of hyperons surrounded by the nucleon liquid component in the plasma, the hyperons are assumed to be localized on the nodes of a cubic lattice under the action of their mutual interaction and the mean fields created by the surrounding nucleons. In this way the total potential at every lattice site is approximated by a harmonic potential which is determined selfconsistently as the superposition of the potentials created by the gaussian clouds of two antiparallel spin hyperons on all the other nodes of the lattice, to which the interaction with the mean field of the nucleon sector is added. In a first approximation we neglect the central and spin-spin interaction between the hyperons in the same node. We shall consider such interaction in future work, but we have already verified that their effect improves the confining character of the whole potential and facilitates the crystallization. In calculating the lattice potential we have also introduced monopolar form factors accounting for the composite structure of the hyperon. In this analysis, vanishing temperature is assumed.

For the nucleonic background in the crystal, a relativistic Hartree approximation is used to calculate the contribution to the total energy density of the system, as in the case of the liquid ground state to which it is compared.

The relative abundances of protons, neutrons and hyperons can be now obtained by establishing the equations of $\beta$-equilibrium between all these particles. The chemical potentials of protons, neutrons and electrons are the Fermi energies of these particles which are in Fermi gaseous phases. The chemical potential of the hyperons are calculated as the energy gained by the system when a new hyperon is added in the $n=1$ level of the harmonic potential in a node. We need also the equations for the mean scalar and vector fields generated by the uniform background of nucleons. The abundance of hyperons resulting from this system is an ingredient in the calculation of the potential created by the lattice on every node.

Consequently, for the periodic configuration the self-consistent set of equa-

bo: submitted to World Scientific on August 19, 2019 
tions for the beta equilibrium of chemical potentials in the plasma coupled to the mean field equations, and the self consistent equations for the confining fields in the nodes are

$$
\begin{aligned}
n_{\text {electrons }} & =n_{\text {protons }} \quad(\text { charge neutrality }) \\
\mu_{\text {neutrons }} & =\mu_{\text {protons }}+\mu_{\text {electrons }} \\
\mu_{\text {neutrons }} & =\mu_{\text {hyperons }} \\
n_{\text {baryons }} & =n_{\text {neutrons }}+n_{\text {protons }}+n_{\text {hyperons }} \\
m_{\sigma}^{2}<\sigma+\sigma_{\text {ext }}> & =g_{\sigma N}<\bar{\Psi} \Psi>+g_{\sigma H} n_{\text {hyperons }} \\
m_{\omega}^{2}<\omega^{0}+\omega_{\text {ext }}^{0}> & =-g_{\omega N}<\bar{\Psi} \gamma^{0} \Psi>-g_{\omega H} n_{\text {hyperons }} \\
m_{\sigma}^{2}<\sigma_{\text {ext }}> & =g_{\sigma H} n_{\text {hyperons }} \\
m_{\omega}^{2}<\omega_{\text {ext }}^{0}> & =-g_{\omega H} n_{\text {hyperons }}
\end{aligned}
$$

The crystal size cell $a$ is related to the hyperonic density $n_{\text {hyperons }}$ of hyperons paired in a S-state.

$$
n_{\text {hyperons }}=\frac{2}{a^{3}}
$$

The solution of this system gives the magnitudes characterizing the configuration, such as crystal cell size, characteristic oscillation frequency and width of hyperon wave functions, the energy levels, etc as functions of the total baryonic density $\mathrm{E}$.

In Figure 1 we show the confining potential in a node of the hyperonic lattice for a set of values of the parameters of the model $(a=2.2142 \mathrm{fm}$, cutoff for the scalar and vector fields, $\Lambda_{\sigma}=1.4 \mathrm{GeV}, \Lambda_{\omega}=2.5 \mathrm{GeV}$, value of the coupling constants $g_{\sigma H} / g_{\sigma N}=0.6, g_{\omega H} / g_{\omega N}=0.65$ taking the Walecka values for the meson-nucleon interaction). This figure has been obtained by the self consistent solution of the equations relating the confining potential to the width of the hyperonic wave function.

Figure 2 shows the cell size in the lattice as a function of baryonic density obtained by asuming the solid configuration to be stable at every baryonic density.

It is worth mentioning that the screening effects of the nucleonic plasma have to be treated properly in order to consider the modification from the screened $\mathrm{HH}$ interaction to the vacuum one, as well as the mixing effects of the hadronic fields. This work is actually in progress.

To determine whether the transition from a spatially uniform hyperonic liquid phase to an ordered one takes place inside the plasma, and the range of thermodynamic variables where the crystal phase is energetically favoured, the free energies of both phases have to be compared. The densities of both 


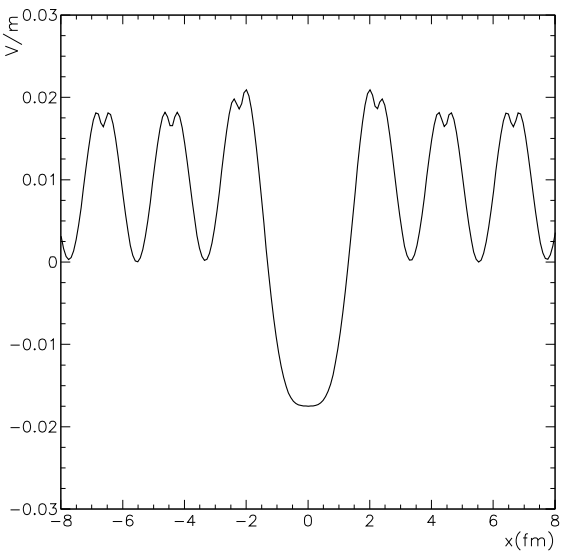

Figure 1. Self consistent confining potential

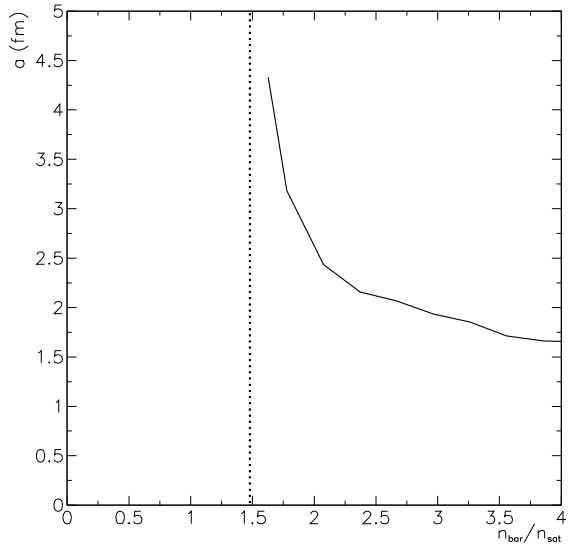

Figure 2. Lattice size as a function of density

phases in the transition region is determined by looking for the cross point between the liquid and solid configuration lines in the diagram of partial hyperon pressure versus hyperonic chemical potential.

This phase transition is of first order, leading to a change in the equation of state of the plasma with a modification of the mechanical properties which should have consequences on the hydrostatic equilibrium of neutron stars and modify the analysis of the cooling processes.

\section{References}

1. M. Prakash, I. Bombaci, M. Prakash, J.M. Lattimer, P. Ellis, R. Knorren, Phys. Rep. 280, 1 (1997).

N.Glendenning, Compact Stars (Springer-Verlag NY, 1997).

2. J.D. Walecka. Ann. Phys. 83, 491 (1974).

3. R. Machleidt, The Meson Theory of Nuclear Forces and Nuclear Structure Adv. in Nucl. Phys. 19, J.W. Negele and E.Vogt eds. (Plenum Press NY, 1989).

4. N.W. Ashcroft, N.D. Mermin, Solid State Physics (Saunders College Publishing, Fort Worth, 1976).

5. J. Díaz Alonso, L. Mornas, M.A. Pérez-García, J.P. Suárez, in preparation.

6. J. Díaz Alonso, A. Pérez Canyellas, Nucl. Phys. A 526, 623 (1991). 